\title{
SOLVING THE SUBSET INTERCONNECTION DESIGN PROBLEM USING GENETIC ALGORITHMS *
}

\author{
Faris N. AbuAli \\ Computer Science Department \\ Yarmouk University \\ Irbid, Jordan
}

\author{
Roger L. Wainwright \\ Dept. of Mathematical and Computer Sciences \\ The University of Tulsa \\ 600 South College Avenue \\ Tulsa, OK 74104-3189, USA \\ rogerw@penguin.mcs.utulsa.edu \\ http://www.utulsa.edu
}

\author{
Dale A. Schoenefeld \\ Dept. of Mathematical and Computer Sciences \\ The University of Tulsa \\ 600 South College Avenue \\ Tulsa, OK 74104-3189, USA \\ dschoen@euler.mes.utulsa.edu \\ http://www.utulsa.edu
}

Key Words: Genetic Algorithms, Interconnection Design Problem, Minimum Spanning Tree

\section{ABSTRACT}

The genetic algorithm (GA) heuristic is used to find near optimal solutions for an NP-complete variation of the minimum spanning tree problem. Given a set of vertices $V$, a cost function $c: V \times V \longrightarrow \Re^{+}$, and a collection of subsets of $V,\left\{X_{1}, \ldots, X_{m}\right\}$, a graph $G$ with vertex set $V$ is called feasible if every $X_{i}$ induces a connected subgraph of $G$. The minimum subset interconnection design problem is to find a feasible graph with a minimum cost. A GA solution is compared to two recently developed approximate solution techniques, and is shown to produce superior results.

\footnotetext{
*Research partially supported by OCAST Grant AR2-004 and Sun Microsystems, Inc.

"Permission to make digital/hard copy of all or part of this material without fee is granted provided that copies are not made or distributed for profit or commercial advantage, the ACM copyright/server notice, the title of the publication and its date appear, and notice is given that copying is by permission of the Association for Computing Machinery, Inc.(ACM). To copy otherwise, to republish, to post on servers or to redistribute to lists, requires prior specific permission and/or a fee"

(C)1996 ACM 0-89791-820-7 9600023.50
}

\section{INTRODUCTION}

The minimum subset interconnection design problem is defined as follows. Given a set of vertices $V$, a cost function $c: V \times V \longrightarrow \Re^{+}$, and a collection of subsets of $V,\left\{X_{1}, \ldots, X_{m}\right\}$, a graph $G$ with vertex set $V$ is called feasible if every $X_{i}$ induces a connected subgraph of $G$. This means that for any two elements $u$ and $v$ in $X_{i}$ there is some $u-v$ path in $G$ consisting only of vertices of $X_{i}$. The minimum subset interconnection design problem is to find a feasible graph with a minimum cost. In this paper we investigate the genetic algorithm (GA) as a heuristic technique for finding optimal or near optimal solutions to this problem.

A wide range of applications can be thought of as interconnection design problems. An example is the design of vacuum systems as presented by Du and Miller[11]. Another example relates to establishing communications networks where some subset of sites needs to be internally connected. Prisner [15] gives an excellent example of communicating networks between employees.

The Interconnection design problem is NP-complete [11]. Special cases of the problem that are not NPcomplete are detailed in[11, 15] and summarized below:

1. When $m$, the number of given subsets, is equal to 1 , the problem reduces to the classical minimum spanning tree problem. 
2. $K(u, v)$, for $u, v \in V$ and $u \neq v$, is the number of subsets, $X_{i}$, containing both vertices $u$ and $v$. When the maximum value of $K(u, v)$ over all $u, v \in V$ is equal to 1 , the problem is solvable in polynomial time.

3. Let $P=$ the maximum value of $\left|X_{i}\right|, i=1, \ldots, m$, where $\left|X_{i}\right|$ is the cardinality of $X_{i}$. When $P=2$, the problem is solvable in polynomial time. If $P \geq 3$ the problem becomes NP-complete.

Prisner [15] recently developed two algorithms for approximately solving the subset interconnection design problem. His first algorithm begins with an edgeless graph $G(V, \Phi)$, and adds new edges consecutively. Edges are chosen based on the total cost-benefit ratio. At every stage of the graph $G$, the edge $v$ - $w$ with maximum $u(v w, G) / c(v w)$ is chosen until this maximum quotient equals zero. The total benefit of edge $v-w$, denoted by $u(v w, G)$, is given by $\sum_{i=1}^{m} u_{i}(v w, G)$, where $u_{i}(v w, G)=0$ if $X_{i}$ does not contain both $v$ and $w$ or if both $v$ and $w$ lie in some common connected component of the actual graph $G\left[X_{i}\right]$, and otherwise, $u_{i}(v w, G)=1$. The cost of edge $v-w$ is denoted by $c(v w)$. We will refer to Prisner's first algorithm as algorithm $\mathrm{P} 1$. The time-complexity of algorithm $\mathrm{P} 1$ is $O\left(n^{4}+m n^{2}\right)$, where $n$ is the number of vertices.

Prisner's second algorithm also constructs the edges, one at a time [15]. First, the algorithm computes the function $K(u, v)$ and the "modified costs" $c(u v) / K(u, v)$ for $u, v \in V$ and $u \neq v$. Next, the algorithm finds a $(c / K)$-minimum spanning tree of $X_{1}$. Then, it completes the already existing edges in $X_{2}$, to obtain a connected spanning subgraph. This is repeated for $X_{3}$, and so on. We will refer to Prisner's second algorithm as algorithm P2. The time-complexity of algorithm P2 is $O\left(n^{2}\left(\log n+m \alpha\left(n^{2}, n\right)\right)\right)$, where $\alpha$ denotes the inverse of the Ackerman function. According to Prisner [15], algorithm P2 seems to be faster than algorithm P1, however algorithm P1 generally gives better results.

For example, in Figure 1, four feasible graphs are shown for a set of 10 vertices and a collection of 10 subsets. The cost matrix and the subsets are shown in Figure 1(a) and Figure 1(b), respectively. Solutions shown in Figure 1(c) and Figure 1(d) were produced using our implementation of Prisner's Algorithms, P1 and P2, respectively. Figure 1(e) and Figure 1(f) show superior solutions from our genetic algorithm, which we describe below.

\section{GENETIC ALGORITHMS}

Genetic Algorithms (GA) are based on the principles of natural genetics and survival of the fittest. Genetic algorithms search for solutions by emulating biological selection and reproduction. In a GA the parameters of the model to be optimized are encoded into a finite length string, usually a string of bits. Each parameter is represented by a portion of the string. The string is called a chromosome, and each bit is called a gene. Each string is given a measure of "fitness" by a fitness function, sometimes called the objective or evaluation function. The fitness of a chromosome determines its ability to survive and reproduce offspring. The "least fit" or weakest chromosomes of the population are displaced by more fit chromosomes. Genetic Algorithms are applicable to a wide variety of problems. In particular, genetic algorithms have been very successful in obtaining near-optimal solutions to many different combinatorial optimization problems $[2,3,4,6,7,9,14,19]$.

Genetic algorithm packages for a single processor have been available for several years. The research reported here made use of LibGA [5], a GA package developed in house. Davis, Goldberg and Rawlins provide an excellent in depth study of genetic algorithms $[6,7,12,16]$. It is assumed the reader is generally familiar with the fundamentals of genetic algorithms.

\section{PROBLEM ENCODING AND EVALUATION FUNCTION}

A graph $G=(V, E)$ can be represented by an adjacency matrix $A$, with entries that are either 0 or 1 . The value of $a_{i j}$ is 1 if there is an edge between $v_{i}$ and $v_{j}$, otherwise $a_{i j}$ is 0 . Our chromosome representation of $G$ is a bit-string corresponding to the upper triangular part of the adjacency matrix in row major order. The length of the chromosome is fixed at $n(n-1) / 2$, where $n$ is the number of vertices in the graph.

To evaluate the fitness of a chromosome, the graph the chromosome represents is checked for feasibility with respect to all of the subsets $X_{i}, 1 \leq i \leq m$, and then the cost of the edges is summed. One difficult feature of the subset interconnection design problem is that the feasibility constraint is a hard constraint rather than a soft constraint. That is, breaking this constraint renders a solution infeasible. Several researchers have developed solutions for the infeasible chromosome problem. The chromosome repair technique used by Liepins et al. [13] and later by Davis et al. [8] is one example. Sekharan et al. [18] developed a technique where the infeasible chromosomes are kept in a separate pool. He probabilistically "mated" chromosomes from the feasible pool with chromosomes from the infeasible pool.

Our algorithm uses the chromosome repair technique when the chromosome is infeasible. This algorithm is described below. The following steps are performed until the chromosome is feasible. 
1. Select a subset $X_{i}$ for $1 \leq i \leq m$.

2. While the chromosome is infeasible with respect to subset $X_{i}$ do steps $3-7$.

3. Select a vertex $u \in X_{i}$ at random.

4. Find the closure $C$ of $u$ in $G$ with respect to $X_{i}$. The closure $C$ of $u$ in $G$ with respect to $X_{i}$ is the set of vertices in $X_{i}$ that can be reached in $G$ from $u$ using only vertices in $X_{i}$.

5. Select a vertex $v \in X_{i}-C$ at random.

6. Add the edge $u-v$ to the graph $G$.

7. Add the cost of the edge $u-v$ to the cost of the graph.

We developed three strategies for the selection of the subsets described in step 1 above:

a) Sequential selection: for $\mathrm{i}=1, \ldots, m$ select subset $X_{i}$.

b) Sorted selection: sort the subsets in increasing order according to cardinality and then do sequential selection.

c) Random selection: randomly pick a permutation $P$, of the $m$ subsets and then select subset $X_{i}$ where $i=P[j]$ for $1 \leq j \leq m$.

Notice that the while loop (steps $3-7$ ) can be rather expensive. However, this allows the algorithm to consider a larger number of potential solutions.

\section{TEST PROBLEMS AND RE- SULTS}

The generational genetic algorithm model was executed using four combinations of single point and uniform crossovers, each with fixed and adaptive mutation rates. The crossover rate is 0.6 . The mutation rate is 0.1 and the selection strategy for the generational GA was roulette (fitness proportionate).

Table 1 shows the results of running algorithms P1 and P2, as developed by Prisner[15], and our genetic algorithm for 10 different random distributions of vertices and subsets. The number of vertices is 30 and the number of subsets is 140 . For all 10 data sets, one random distribution of vertices is generated, and the Euclidean distance between the vertices is used as the cost of connecting the vertices. Then, for each data set, a random distribution of subsets was produced. The size of each subset was randomly selected in the range from 2 to the number of vertices.

In each of the 10 data sets tested, the generational genetic algorithm with uniform crossover and adaptive mutation rate outperformed algorithms $\mathrm{P} 1$ and P2. Notice that the GA using adaptive mutation consistently performed better than fixed mutation regardless of the crossover method used.

Table 2 shows the results for a total of 34 runs on eight different sets of vertices. The cardinalities of the eight sets of vertices range from $n=5$ to $n=40$ in increments of 5. For each set $V$ of vertices, four different collections of subsets of $V$ were generated. The first collection of subsets contained $1^{*}|V|$ subsets of $V$. Similarly, the second, third and fourth collections of subsets of $V$ contained $2^{*}|V|, 3^{*}|V|$, and $4^{*}|V|$ subsets of $V$, respectively. In addition, for the case where $|V|=35$, a collection of $8^{*} 35=280$ subsets of $V$ was generated and tested. Similarly, for the case where $|V|=40$, a collection of $8^{*} 40=320$ subsets of $V$ was generated and tested. In each case, the size of each subset was randomly generated in the range from 2 to $|V|$, the number of vertices.

In all 34 cases tested, at least one of our genetic algorithms outperformed algorithm P2. In 28 of the 34 cases, at least one of our genetic algorithms produced the same or better results than algorithm P1. In the remaining 6 cases, all occurring when $n$ is large and the number of subsets is relatively small, the GA did not do as well as algorithm P1. On the other hand, when the number of subsets is increased, the GA consistently produced better results.

\section{CONCLUSION}

To the authors' knowledge a genetic algorithm implementation for the subset interconnection design problem has not been implemented before. Prisner [15] has recently developed two near optimal algorithms for this problem. We developed a genetic algorithm for the subset interconnection design problem and tested it on a variety of problems comparing our results to Prisner's algorithms. Our GA exhibited superior results in nearly all of the cases tested. We determined that our best GA to solve this problem uses uniform crossover with adaptive mutation. Our three selection strategies to sequence the selection of the subsets in order to convert an infeasible chromosome to a feasible chromosome were indistinguishable in their results. No one algorithm appeared to be any better than the others. Prisner's algorithms run in the order of minutes and our GA runs in the order of hours. Because the GA yields superior results in most cases, we recommend that researchers run the GA as well as Prisner's algorithms to obtain the best result possible. 


\begin{tabular}{|c|c|c|c|c|c|c|c|c|c|c|c|}
\hline \multirow{2}{*}{\multicolumn{2}{|c|}{ Algorithm }} & \multicolumn{10}{|c|}{ Random data sets } \\
\hline & & $\mathrm{I}$ & II & III & IV & $\mathrm{V}$ & VI & VII & VIII & IX & $\mathrm{X}$ \\
\hline \multicolumn{2}{|c|}{ Algorithm P1 } & 3706 & 4452 & 4247 & 4093 & 4010 & 4179 & 4212 & 4216 & 3621 & 4233 \\
\hline & n P2 & 4703 & 5162 & 5206 & 4817 & 4880 & 5018 & 4820 & 4858 & 4337 & 5069 \\
\hline \multicolumn{2}{|c|}{ Genetic Alg. } & \multirow[b]{3}{*}{3749} & \multirow[b]{3}{*}{$4188^{*}$} & \multirow[b]{3}{*}{4482} & \multirow[b]{3}{*}{$3846^{*}$} & \multirow[b]{3}{*}{4070} & \multirow[b]{3}{*}{3874} & \multirow[b]{3}{*}{4178} & \multirow[b]{3}{*}{4186} & \multirow[b]{3}{*}{$3393^{*}$} & \multirow[b]{3}{*}{4428} \\
\hline Crossover & Mut. & & & & & & & & & & \\
\hline Single pt & Adapt & & & & & & & & & & \\
\hline Uniform & Adapt & $3623^{*}$ & 4190 & $4104^{*}$ & 3851 & $3749^{*}$ & $3826^{*}$ & $3968^{*}$ & $4160^{*}$ & 3559 & $4003^{*}$ \\
\hline Single pt & Fixed & 4107 & 4628 & 4755 & 4624 & 4608 & 4855 & 4771 & 4757 & 4450 & 4994 \\
\hline Uniform & Fixed & 3986 & 4535 & 4502 & 4089 & 4156 & 4251 & 4627 & 4340 & 3787 & 4481 \\
\hline
\end{tabular}

Table 1: Subset Interconnection Design problem for 30 nodes and 140 subsets. A “*” indicates the best.

\section{Acknowledgements}

This research has been supported by OCAST Grant AR2-004. The authors would like to thank Referee No. 4 for his thorough reading of the manuscript, which resulted in numerous improvements and clarifications. We gratefully acknowledge the support of Sun Microsystems, Inc.

\section{References}

[1] Hal Berghel, Ed Deaton, George Hedrick, David Roach, and Roger Wainwright, editors. Proceedings of the 1992 ACM/SIGAPP Symposium on Applied Computing, New York, 1992. ACM Press.

[2] J. L. Blanton and R. L. Wainwright. Vehicle routing with time windows using genetic algorithms. In William A. Coberly, editor, Proceedings of the Sixth Oklahoma Symposium on Artificial Intelligence, pages 242-251, Tulsa, Oklahoma, November 1992.

[3] D. E. Brown, C. L. Huntley, and A. R. Spillane. A parallel genetic heuristic for the quadratic assignment problem. In Schaffer [17].

[4] A. L. Corcoran and R. L. Wainwright. A genetic algorithm for packing in three dimensions. In Berghel et al. [1], pages 1021-1030.

[5] A. L. Corcoran and R. L. Wainwright. LibGA: A user-friendly workbench for order-based genetic algorithm research. In Deaton et al. [10], pages 111118.

[6] L. Davis, editor. Genetic Algorithms and Simulated Annealing. Morgan Kaufmann, 1987.

[7] L. Davis, editor. Handbook of Genetic Algorithms. Van Nostrand Reinhold, New York, 1991.

[8] Lawrence Davis, David Orvosh, Anthony Cox, and Yuping Qiu. A genetic algorithm for survivable network design. In Stephanie Forrest, editor, Proceedings of the Fifth International Conference on
Genetic Algorithms, Urbana-Champaign, Illinois, July 1993. Morgan Kaufmann.

[9] Kenneth A. De Jong and W. M. Spears. Using genetic algorithms to solve np-complete problems. In Schaffer [17], pages 124-132.

[10] Ed Deaton, K. M. George, Hal Berghel, and George Hedrick, editors. Proceedings of the 1993 ACM/SIGAPP Symposium on Applied Computing, New York, 1993. ACM Press.

[11] D-Z. Du and Z. Miller. Matroids and subset interconnection design. SIAM J. Discrete Math, 1(4):416-424, 1988

[12] David E. Goldberg. Genetic Algorithms in Search, Optimization, and Machine Learning. AddisonWesley, Reading, Massachusetts, 1989.

[13] G. E. Liepins, M. R. Hilliard, J. Richardson, and M. Palmer. Genetic algorithm applications to set covering and traveling salesman problems. In Brown, editor, OR/AI: The Integration of Problem Solving Strategies. 1990.

[14] P. P. Mutalik, L. R. Knight, J. L. Blanton, and R. L. Wainwright. Solving combinatorial optimization problems using parallel simulated annealing and parallel genetic algorithms. In Berghel et al. [1], pages 1031-1038.

[15] Erich Prisner. Two algorithms for the subset interconnection design problem. Networks, 22:385-395, 1992.

[16] G. Rawlins, editor. Foundations of Genetic Algorithms. Morgan Kaufmann, 1991.

[17] J. David Schaffer, editor. Proceedings of the Third International Conference on Genetic Algorithms, Arlington, Virginia, 1989. Morgan Kaufmann.

[18] D. Ansa Sekharan and Roger L. Wainwright. Manipulating subpopulations of feasible and infeasible solutions in genetic algorithms. In Deaton et al. [10], pages $118-125$. 


\begin{tabular}{|c|c|c|c|c|c|c|}
\hline \multirow{2}{*}{$\begin{array}{c}\text { Number of } \\
\text { Vertices }\end{array}$} & \multirow{2}{*}{$\begin{array}{c}\text { Number of } \\
\text { Subsets }\end{array}$} & \multirow[t]{2}{*}{ Algorithm P1 } & \multirow[t]{2}{*}{ Algorithm P2 } & \multicolumn{3}{|c|}{ Genetic Algorithm } \\
\hline & & & & Random & Sequential & Sorted \\
\hline \multirow{4}{*}{5} & 5 & $196^{*}$ & $196^{*}$ & $196^{*}$ & $196^{*}$ & $196^{*}$ \\
\hline & 10 & $241^{*}$ & $241^{*}$ & $241^{*}$ & $241^{*}$ & $241^{*}$ \\
\hline & 15 & $281^{*}$ & $281^{*}$ & $281^{*}$ & $281^{*}$ & $281^{*}$ \\
\hline & 20 & $332^{*}$ & 334 & $332^{*}$ & $332^{*}$ & $332^{*}$ \\
\hline \multirow{4}{*}{10} & 10 & 534 & 525 & $486^{*}$ & $486^{*}$ & 503 \\
\hline & 20 & 799 & 887 & $725^{*}$ & 736 & $725^{*}$ \\
\hline & 30 & 954 & 1103 & 904 & $896^{*}$ & $896^{*}$ \\
\hline & 40 & 976 & 1165 & $925^{*}$ & $925^{*}$ & $925^{*}$ \\
\hline \multirow{4}{*}{15} & 15 & 785 & 766 & $678^{*}$ & $678^{*}$ & 703 \\
\hline & 30 & 1106 & 1201 & 955 & 964 & $946^{*}$ \\
\hline & 45 & 1417 & 1600 & 1295 & 1285 & $1269^{*}$ \\
\hline & 60 & 1651 & 1898 & $1525^{*}$ & 1566 & 1528 \\
\hline \multirow{4}{*}{20} & 20 & 1396 & 1480 & 1239 & $1177^{*}$ & 1265 \\
\hline & 40 & 1901 & 2032 & 1792 & $1720^{*}$ & 1853 \\
\hline & 60 & 2406 & 2811 & 2187 & 2309 & $2155^{*}$ \\
\hline & 80 & 2891 & 3169 & 2650 & $2580^{*}$ & 2620 \\
\hline \multirow{4}{*}{25} & 25 & 1590 & 1819 & 1590 & 1615 & $1524^{*}$ \\
\hline & 50 & 2141 & 2535 & $2031^{*}$ & 2188 & 2098 \\
\hline & 75 & 2493 & 3036 & 2504 & 2460 & $2393^{*}$ \\
\hline & 100 & 2848 & 3706 & 2770 & $2696^{*}$ & 2711 \\
\hline \multirow{4}{*}{30} & 30 & 2056 & 2303 & 2094 & 2115 & $1905^{*}$ \\
\hline & 60 & 2891 & 3268 & $2627^{*}$ & 2639 & 2671 \\
\hline & 90 & 3382 & 3712 & 3182 & $2971^{*}$ & 3073 \\
\hline & 120 & 3891 & 4883 & $3509^{*}$ & 3630 & 3569 \\
\hline \multirow{5}{*}{35} & 35 & $2294^{*}$ & 2869 & 2423 & 2485 & 2418 \\
\hline & 70 & $3202^{*}$ & 3853 & 3324 & 3264 & 3265 \\
\hline & 105 & 3889 & 4887 & 3894 & 4091 & $3807^{*}$ \\
\hline & 140 & $4346^{*}$ & 5178 & 4401 & 4444 & 4577 \\
\hline & 280 & 6013 & 8421 & $5906^{*}$ & 6028 & 6020 \\
\hline \multirow{5}{*}{40} & 40 & $2558^{*}$ & 2984 & 2930 & 3294 & 2978 \\
\hline & 80 & $3461^{*}$ & 4643 & 3952 & 3887 & 3810 \\
\hline & 120 & $4246^{*}$ & 5526 & 4851 & 4759 & 4773 \\
\hline & 160 & 5143 & 6413 & $4908^{*}$ & 5417 & 5113 \\
\hline & 320 & 7121 & 10189 & 7143 & $6890^{*}$ & 6941 \\
\hline
\end{tabular}

Table 2: Subset Interconnection Design problem using Genetic Algorithms with uniform crossover, crossover rate 0.6 , and adaptive mutation. A “*” indicates the best.

[19] Darrell Whitley, T. Starkweather, and D. Fuquay. Scheduling problems and traveling salesman: The genetic edge recombination operator. In Schaffer [17]. 


\begin{tabular}{c|cccccccccc}
\multicolumn{10}{c}{ Cost matrix } \\
& 1 & 2 & 3 & 4 & 5 & 6 & 7 & 8 & 9 & 10 \\
\hline 1 & $*$ & 71 & 45 & 46 & 43 & 26 & 43 & 49 & 31 & 54 \\
2 & & $*$ & 73 & 97 & 59 & 66 & 114 & 56 & 69 & 125 \\
3 & & & $*$ & 91 & 14 & 68 & 74 & 84 & 14 & 75 \\
4 & & & & $*$ & 88 & 32 & 46 & 47 & 77 & 63 \\
5 & & & & & $*$ & 61 & 78 & 74 & 16 & 82 \\
6 & & & & & & $*$ & 56 & 24 & 54 & 71 \\
7 & & & & & & & $*$ & 80 & 62 & 17 \\
8 & & & & & & & & $*$ & 71 & 96 \\
9 & & & & & & & & & $*$ & 66 \\
10 & & & & & & & & & & $*$
\end{tabular}

(a)

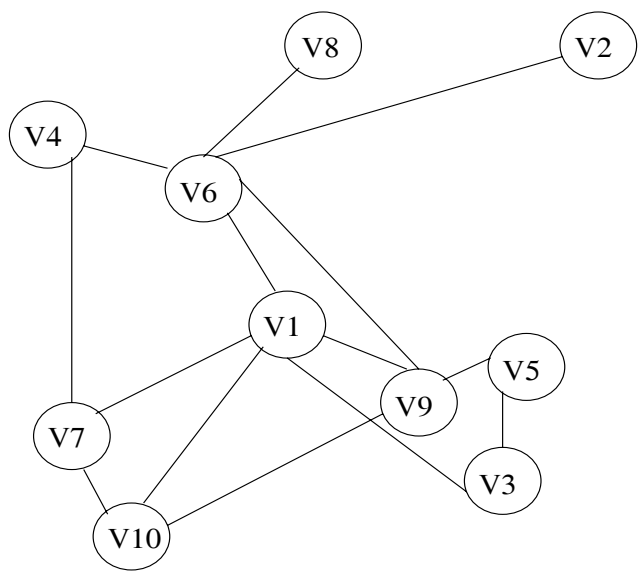

(c)

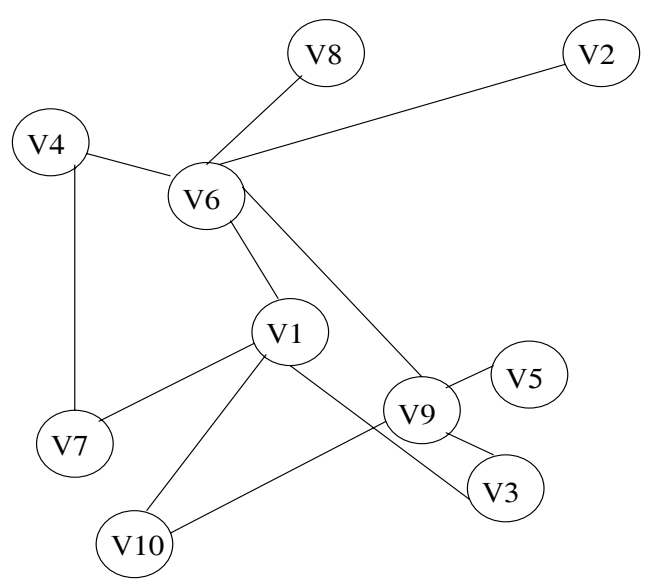

(e)

\begin{tabular}{r|rrrrrrr}
\multicolumn{7}{c}{ Subsets of $V$} \\
\multicolumn{1}{c|}{} & \multicolumn{7}{c}{$X_{i}$} \\
2 & 1 & 4 & 5 & 6 & 9 & 10 & \\
3 & 2 & 4 & 5 & 6 & 7 & 9 & 10 \\
4 & 1 & 2 & 5 & 6 & 8 & 9 & 10 \\
5 & 1 & 3 & 6 & 8 & & & \\
6 & 1 & 2 & 4 & 6 & 8 & 9 & 10 \\
7 & 1 & 3 & 7 & & & & \\
8 & 1 & 10 & & & & & \\
9 & 6 & 9 & 10 & & & & \\
10 & 1 & 3 & 4 & 5 & 7 & 9 &
\end{tabular}

(b)

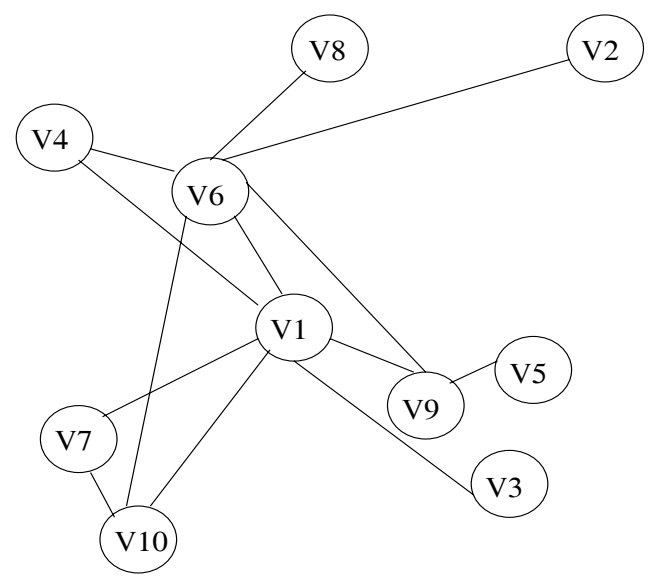

(d)

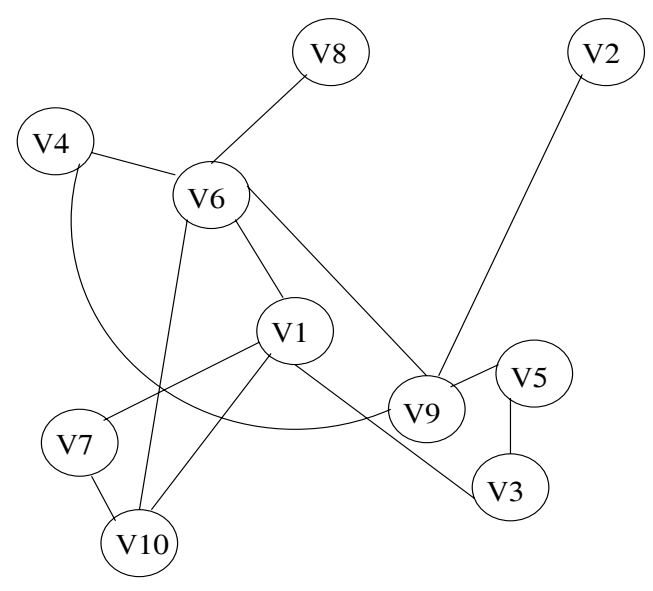

(f)

Figure 1: Subset Interconnection Design problem for 10 nodes and 10 subsets. (a) The cost matrix, (b) The subsets of $V$, (c) Algorithm P1 with a cost of 534, (d) Algorithm P2 with a cost of 525, (e) Genetic Algorithm with uniform crossover with a cost of 486, (f) Genetic Algorithm with single point crossover with a cost of 510. 